\title{
The technology for automated development of economically doped heat-resistant nickel superalloys
}

\author{
Yuriy Shmotin, Aleksander Logunov, Denis Danilov, and Igor Leshchenkoi ${ }^{\mathrm{a}}$ \\ JSC “NPO SATURN”, 163 Lenin avenue, Rybinsk 152903, Russia
}

\begin{abstract}
The paper presents the usage of computational technology, aimed at automated search for optimum compositions of nickel superalloys, being minimally doped by expensive rhenium and having no ruthenium. Resulting doping compositions ensure ultimate long-term high-temperature strength. The technology is based on computer model of alloy and numerical optimization routine. The model computes relation between an alloy composition and its performance characteristics. Multiobjective optimization procedure provides for an automated search for alloy composition to ensure the set of pre-defined properties of a new material. Two new nickel-based single-crystal superalloys are presented, which were created using this technology. SLGS-3 features high-temperature strength (100 hours at 1,000 degrees Centigrade) at approximately $345 \mathrm{MPa}$. Its composition does not contain ruthenium. SLGS-1 alloy provided for $265 \mathrm{MPa}$ and contained neither rhenium nor ruthenium in its composition.
\end{abstract}

\section{Introduction}

Potential of development of new gas turbine engines is directly connected with availability of high performance heat-resistant superalloys for turbine blades. Doping nickel alloys with rhenium and ruthenium improves their performance characteristics. Application of these elements resulted in creating nickel-based materials of the 4th generation (MS-NG - France, TMS-162 and TMS-196 - Japan, EPM-102 - USA, etc.). However the price of these elements is extremely high and it continues to grow. So, the abovementioned direction of developing new hightemperature superalloys can become not economically viable if the price of doping elements rises too high.

Accordingly, it is expedient to develop alloys comparable in terms of their heat-resistance characteristics to the present-day single-crystal superalloys, that could be produced with at least one of the above high-cost alloying elements, if none at all.

To get the composition of highly effective and economically doped alloy, one needs development and application of special methods. Firstly, it is required to perform quick, inexpensive and authentic evaluation and analysis of large number of thermodynamic, structural, strength, concentration, kinetic, physical and chemical parameters. Special computer models must be created to get the information on these parameters. On the other hand, for solving the problem it is not enough to have an adequate model only, because it is necessary to coordinate optimally about dozen of independent variables - doping elements weight fractions. From the mathematical point of view it is an uneasy task.

\footnotetext{
${ }^{a}$ Corresponding author: igor.leshchenko@yandex.ru
}

Therefore the problem of development of highperformance heat resisting nickel alloys can be successfully solved only on the basis of a combination of their computer modelling with procedure of numerical optimization of their composition.

The present work represents the implementation of computational technology, used for obtaining the compositions of alloys, having the defined set of properties. The components of this technology are mathematical model, optimization procedure and optimization task statement.

\section{Criteria of alloy quality estimation}

The prominent feature of modern period of development in the field of materials and technologies is considerable complication of both alloys compositions and processes of manufacturing engine details, having special characteristics. When manufacturing the single crystal rotor and stator cooled blades, it is required to consider a great number of factors, connected with ensuring the perfect structure of material, in which there are no harmful phase formations, cavities and porosities; as well as with necessity of applying the effective coatings which must reliably protect the details of the engine from high-temperature corrosive attack during all term of their operation.

There is one more essential factor complicating the work, aimed at creation high temperature superalloys. It is connected with the fact that areas of optimum multicomponent doping have become extremely close to the spheres of existence of brittle topologically close packed (TCP) compounds. Therefore the admissible intervals of doping elements contents were considerably narrowed owing to necessity of ensuring the guaranteed absence of harmful phases in alloy structure.

This is an Open Access article distributed under the terms of the Creative Commons Attribution License 4.0, which permits unrestricted use, distribution, and reproduction in any medium, provided the original work is properly cited. 
Thus, the reached complexity of doping has led to the fact that further development and optimization of perspective compositions of heat resisting nickel alloys are continuously connected with simultaneous taking into account a significant number of factors, directly influencing the working capacity. Besides a comprehensive estimation of the standard indicators of efficiency of such alloys, it is absolutely necessary to work out the new criteria for estimating the stability of gamma-solid solution from the positions of possible formation of brittle TCP phases.

At present work the following groups of parameters, reflecting the working capacity of heat resisting alloys, were taken into consideration during the research, aimed at obtaining their optimum composition:

1. Strength parameters

- $\sigma_{\tau}^{t}-$ long term high temperature creep rupture strength of the alloy, at temperature $t,{ }^{\circ} \mathrm{C}$, during $\tau$ hours.

2. Thermodynamic parameters

- $T_{B D}$ - temperature of beginning of $\gamma^{\prime}$ - phase dissolution;

- $T_{\text {Solvus }}$ - temperature of complete dissolution of $\gamma^{\prime}$ - phase;

- $T_{S}, T_{L}$ - temperatures solidus and liquidus;

- etc.

3. Structural factors

- Lattice misfit - a relative difference of parameters of crystal lattices in $\gamma$ and $\gamma^{\prime}$ phases (according to [1])

$$
\left(\frac{\Delta a_{\gamma-\gamma^{\prime}}}{a_{\gamma}}=\frac{a_{\gamma}-a_{\gamma^{\prime}}}{0.5 \cdot\left(a_{\gamma}+a_{\gamma^{\prime}}\right)} \cdot 100 \%\right),
$$

where $a_{\gamma}$ and $a_{\gamma^{\prime}}-$ parameters of crystal lattices in $\gamma$ and $\gamma^{\prime}$ phases respectively.

- $V_{\gamma^{\prime}}$ - a volume fraction of $\gamma^{\prime}$ phases;

- $M(\bar{d})_{\gamma}$ - the parameter characterizing the level of concentration of valence electrons of $\gamma$-phase; it presents the probability of forming the brittle TCP compounds (according to method New PHACOMP [2]); if $M(\bar{d})_{\gamma}>$ 0.93 , TCP compounds are highly probable according to [3];

4. Concentration parameters, characterizing the probability of laminated compounds formation, based on $\mathrm{Ni}_{3} \mathrm{Ti}, \mathrm{Ni} i_{3} \mathrm{Nb}, \mathrm{Ni} i_{3} \mathrm{Ta}$, etc., inside the $\gamma^{\prime}$ phases:

$$
-\frac{m_{A l}}{m_{T i}}, \frac{m_{A l}}{m_{N b}+m_{T a}}, \frac{m_{A l}}{m_{T i}+m_{N b}+m_{T a}} ;
$$

5. The parameters characterizing resistance to sulfideoxide corrosion:

$$
-\frac{m_{C r}}{m_{A l}}, \frac{m_{A l}}{m_{T i} \cdot \sqrt{m_{C r}}} \text {. }
$$

In addition to the above listed parameters of alloys, the new concentration criterion was entered - volume fraction of $(A l+T i+W+M o+R e+T a+N b+R u)$ in $\gamma$ phase, which should not exceed $30 \%$.

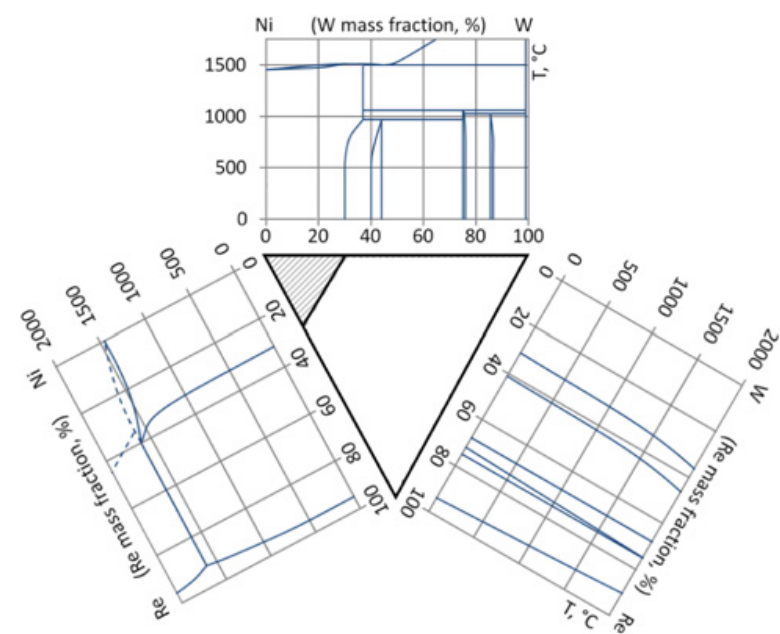

Figure 1. Ternary Ni-Re-W diagram - hatched area presents stable $\gamma$-phase at working temperatures.

Necessity of introduction of new criterion is substantiated by considerable complication of doping owing to possible formation of undesirable phases. The essence of this criterion could be explained by means of Fig. 1, where the part of threefold diagram $N i-W-R e$ is shown. This diagram seems to be most convenient for analysis, because, perhaps, it is the best illustration of situation in $\gamma$ - solid solution which is formed in the alloy. This solution is comprised by $\mathrm{Ni}$, which is the basis of $\gamma$ - matrix, and also $W$ and $\operatorname{Re}(R u)$. Co and $\mathrm{Cr}$, forming with $\mathrm{Ni}$ continuous solid solutions, settle down in this diagram as though together with $\mathrm{Ni}$. As to other elements entering in $\gamma$ - solid solution $(A l, M o, T i, N b, H f$, etc. $)$, they are present at it in small quantities and their total concentration narrows area of homogeneity of a solid solution on the basis of $\mathrm{Ni}$ (on the same size as a first approximation).

The analysis of threefold diagram $N i-W-R e$ shows that total possible concentration of $R e$ and $W$ (and also other elements compounding the $\gamma$ - solid solution $-A l$, $\mathrm{Mo}, \mathrm{Ti}, \mathrm{Nb}, \mathrm{Hf}, \mathrm{Ru}, \mathrm{Ta}$, etc.) should not exceed $30 \%$ by mass, since otherwise the undesirable phases will be allocated from $\gamma$-phases, such as $N i_{4} W$ ( $\beta$-phases), $N i W$ ( $\delta$-phases), $W_{2} \operatorname{Re}_{3}$ ( $\sigma$-phases), $\alpha-R e$ etc, including the combinations of these phases. Such allocation causes additional embrittlement of alloys, and, except that, a leaving from $\gamma$-matrixes of the elements, making decisive contribution into high-temperature hardening of this phase.

All above mentioned parameters should be considered during carrying out the research. The mathematical model is necessary for this purpose.

\section{Mathematical model for calculation the characteristics of heat resistant alloys}

The mathematical model (MM) represents the program code uniting various approaches for evaluating operational properties of an alloy. The MM receives alloy composition as input data, and produces as the result its operational indices. 
As the input data MM uses mass fractions of all doping components of alloy. In total 11 chemical elements are considered: $\mathrm{Cr}, \mathrm{Co}, \mathrm{W}, \mathrm{Mo}, \mathrm{Al}, \mathrm{Ti}, \mathrm{Ta}, \mathrm{Re}, \mathrm{Nb}, \mathrm{Hf}, \mathrm{Ru}$.

The MM computes properties by means of 2 methods. For those parameters which are defined directly in experiment, there are used the response surface models (RSM). The RSM are constructed using databases on existing nickel superalloys, which are being continuously supplemented by data, published in scientific papers. At present such parameters are thermodynamic and strength characteristics, and also:

$V_{\gamma^{\prime}}$ - part by volume of $\gamma^{\prime}$-phase;

$d$ - alloy density;

$K_{C r}, K_{C o}, K_{W}, K_{M o}, K_{A l}, K_{T i}, K_{T a}, K_{R e}, K_{N b}-$ distribution coefficients (volume part of individual element in $\gamma^{\prime}$-phase divided by that in $\gamma$-phase).

Values of $M(\bar{d})_{\gamma}$ and lattice misfit are determined by means of calculations, using known expressions ([1, 2]) and basing on alloy composition and indices, obtained by RSM.

\section{Peculiarities of RSM construction}

\subsection{Approximation techniques}

The key moment at construction of heat resisting alloy mathematical model is ensuring of high reliability of approximating functions, which depend on a structure of RSM. In the literature (for example, [4]) there are described the models based on linear regression, where parameters of alloy properties are defined as:

$$
\text { Par }=R_{0}+\sum_{i=1, N_{\text {Comp }}} R_{i} \cdot m_{i},
$$

where $R_{0}, R_{i}$ - regression coefficients,

$m_{i}$ - alloy doping elements mass fractions,

$N_{\text {Comp }}$ - number of alloy doping elements.

Doubtless advantages of linear regression are simplicity of algorithm of coefficients (factors) determining, rather small quantity of points, necessary for procedure work, and also the possibility of obtaining of functional dependence as analytical expression. However, the more nonlinearity presents in actual dependence between the approximated parameter and arguments, the worse is accuracy of such approach.

Within the frameworks of this work authors were not limited to linear regression at construction of heat resisting alloy model. In addition, following algorithmic implementations of techniques for RSM construction were considered:

- Modified least squares method with expanded set of argument variables. In the basis of this approach it is used the full-square regression with expanded set of variables, forming regressors. Not only mass fractions of doping elements themselves $\left(m_{i}\right)$ comprise the extended set, but also functional dependencies of these variables $\left(\frac{1}{m_{i}}, e^{m_{i}}\right.$, etc.). The complete number of regressors can appear very big, therefore the given algorithm applies adaptive selection of only those regressors, which are most informative. Available option of this type of approximation procedure is the maximum number of regressors, which must be less than the number of source data points. Increasing the number of regressors improves the accuracy of source data mathematical description, but the approximation function becomes less smooth.

- Weighted approximation. For a given source data set of $m$ points $\left(\overrightarrow{\mathbf{x}}^{1}, \overrightarrow{\mathbf{x}}^{2}, \overrightarrow{\mathbf{x}}^{3}, \ldots \overrightarrow{\mathbf{x}}^{m}\right)$ approximation function is defined as:

$$
f(\overrightarrow{\mathbf{x}})=\frac{\sum_{j=1}^{m} W^{j}(\overrightarrow{\mathbf{x}}) \cdot f\left(\overrightarrow{\mathbf{x}}^{j}\right)}{\sum_{j=1}^{m} W^{j}(\overrightarrow{\mathbf{x}})},
$$

where $\overrightarrow{\mathbf{x}}^{j}-j$-th point (vector) of source data set;

$f\left(\overrightarrow{\mathbf{x}}^{j}\right)$ - function value in $j$-th point;

$W^{j}(\overrightarrow{\mathbf{x}})$ - weight function of $j$-th point.

Weight function $W^{j}(\overrightarrow{\mathbf{x}})$ represents the function, which value is equal 1 at $\overrightarrow{\mathbf{x}}=\overrightarrow{\mathbf{x}}^{j}$, and tends to reduce when increasing the distance from point $\overrightarrow{\mathbf{x}}$ to $\overrightarrow{\mathbf{x}}^{j}$. The parameter of flexibility of weighted approximation defines how strongly the weight of point $\overrightarrow{\mathbf{x}}^{j}$ is reduced at distancing $\overrightarrow{\mathbf{x}}$ from it. The more strongly the weight decreases, the more close to source points is the approximation function, however thus it becomes less smooth.

- Regression with local weighting. In the literature [5] the given approach is presented as "Moving Least Squares". The basic idea of the approach consists in improvement of accuracy of linear regression for the description of each point of space at the expense of adaptive application of weight factors to source data points, used to build regression. The weight of each source point is defined individually according to weight function, which decreases when the distance between the source and approximated points is growing. Unlike traditional regression, the method of the least squares is applied each time the approximation function is called.

\section{Accuracy of approximation and prediction}

It is obvious that for effective search of new alloy composition, satisfying the set of requirements, it is necessary to use mathematical model, being accurate as much as possible. When using the RSM, the accuracy estimation is usually done by comparison of source values of the approximated parameter with those values, computed by approximation function. The values of mean error or maximum error, evaluated as mentioned above for different approximation procedures, are usually used for comparison of their accuracy on source data sets. Let's name these indicators of accuracy "approximating".

When one uses the experimental results on existing alloys as the source data, there is a feature connected with limited number of source points. In this case the moving towards the raise of approximation accuracy of source data by means of corresponding settings of RSM procedures options, as a rule, turns into appreciable deterioration of accuracy of approximated parameter evaluation in the areas of space, being a little far from source points. 


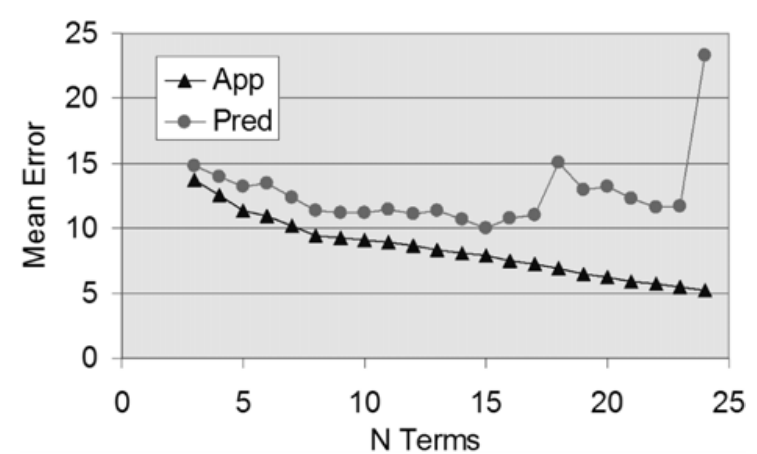

Figure 2. Mean error for $\sigma_{100}^{1000}$ depending on number of regressors.

To get the quantitative assessment of accuracy, which can be achieved in predicting the functional dependence in the areas, where there are no source points, the following procedure is offered. The set of source points is divided into not crossed training and checking subsets. Approximation function is constructed on the training subset. When it is being built, there is no information on the values of approximated parameter in the points of checking subset. Hence, the RSM error on points of a checking subset can be used as an estimation of its predictive ability.

In this work following approach is applied. Only one source point is excluded from training subset, and predicting error is evaluated for it. The cycle is carried out, in which all source points are used as checking ones. The mean error and maximum error are computed with use of all source points, providing that no of these points are used for construction of approximation function. These errors are the indicators, characterizing ability of the RSM to predict the values of function for areas, where it is lack of source points. Let's name these indicators of accuracy "predictive".

Improvement of approximating accuracy by means of tuning the options of RSM procedure allows improving the predictive accuracy indicators only in some limits. For example, Fig. 1 demonstrates testing of approximation procedure for long durability $\sigma_{100}^{1000}$, which experimental database was presented by 51 source points. It is shown, that increasing the number of regressors for the technique "Modified least squares method with expanded set of argument variables" continuously improves the approximating indicators. In an utmost case, when the number of regressors is equal to that of source points, the exact reproduction is probable. At the same time for the number of terms (regressors) greater than 15 , the deterioration of predictive accuracy is observed. It is connected with redundancy of the information which is given by 16-th and the subsequent terms.

Similar "optimum" on predictive accuracy, as a rule, is observed for other techniques of RSM construction.

Thus, when constructing the models of alloy properties, it is necessary to carry out the comparative analysis of approximating and predictive levels of accuracy for various techniques of RSM construction, using different options.
Table 1. RSM Accuracy Indices List.

\begin{tabular}{|c|c|c|c|c|c|}
\hline  & 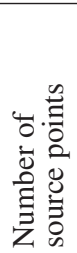 &  & 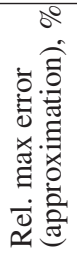 & 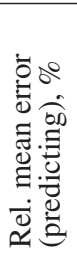 & 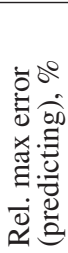 \\
\hline$V_{\gamma^{\prime}}$ & 108 & 4.1 & 10.3 & 4.9 & 12.2 \\
\hline$T_{\text {Solvus }}$ & 109 & 4.4 & 11.8 & 5.3 & 12.8 \\
\hline$\sigma_{100}^{1000}$ & 93 & 3.8 & 11.2 & 4.6 & 12.7 \\
\hline$K_{i C r}$ & 49 & 3.7 & 9.2 & 5.4 & 11.8 \\
\hline$K_{i \text { Co }}$ & 48 & 9.1 & 25.2 & 11.4 & 28.2 \\
\hline$K_{i W}$ & 43 & 7.8 & 20.0 & 12.7 & 38.7 \\
\hline$K_{i M o}$ & 45 & 6.7 & 21.8 & 9.4 & 31.3 \\
\hline$K_{i A l}$ & 49 & 2.5 & 8.0 & 5.9 & 20.8 \\
\hline$K_{i T i}$ & 41 & 3.9 & 9.2 & 8.4 & 25.6 \\
\hline$K_{i T a}$ & 26 & 11.7 & 50.0 & 26.1 & 83.4 \\
\hline$K_{i R e}$ & 16 & 2.7 & 5.8 & 5.0 & 10.3 \\
\hline$K_{i N b}$ & 16 & 5.9 & 13.4 & 28.2 & 69.2 \\
\hline
\end{tabular}
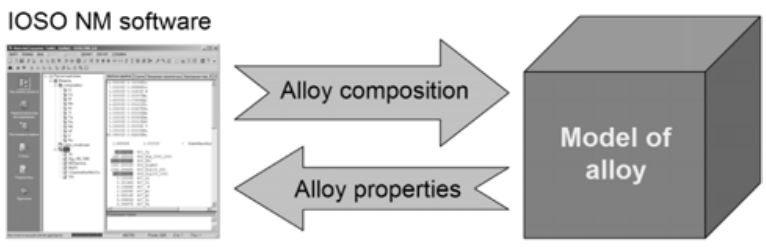

Figure 3. The structure of optimization model.

\section{Results of researches for choice the best function of approximation}

Research aimed at choice of best setting of RSM must be carried out for each of approximated parameters, used in optimization search. The dependences similar to shown on Fig. 1 were used to choose such options of RSM, which provided the least level of predictive error. Results of RSM accuracy estimation for up-to-date state of source database are listed in Table 1.

The level of predictive mean error of long-term strength index of an order of $5 \%$ can be considered as satisfactory for use in basic researches.

\section{Optimization task statement}

According to the purposes of the present research the optimization model has been developed. Its structure includes the mathematical model of heat resisting alloy, presented above, and also the procedure of multiobjective optimization, implemented in IOSO NM software complex [6]. The structure of optimization model is shown in Fig. 3.

Optimization procedure sets the composition of alloy and launches the MM of alloy, which returns its properties. During the optimization such compositions are found, which provide the extremely achievable values of objectives, depending on alloy efficiency indices. Following optimization task formal statement was used.

Independent design variables: mass fractions of 10 doping components ( $\mathrm{Cr}, \mathrm{Co}, \mathrm{W}, \mathrm{Mo}, \mathrm{Al}, \mathrm{Ti}, \mathrm{Ta}, \mathrm{Re}, \mathrm{Nb}$, $H f)$. Objective functions: 
Table 2. Nominal composition of alloys: Mass fractions of elements.

\begin{tabular}{|c|c|c|c|c|c|c|c|c|c|c|c|}
\hline Alloy & Cr & Co & W & Mo & Al & Ti & Ta & Re & Nb & Hf & Ni \\
\hline SLGS-1 & 5 & 10 & 11.5 & 0 & 5 & 0 & 9 & 0.1 & 0 & 0 & Bal \\
\hline SLGS-3 & 3.8 & 3.5 & 8.3 & 0 & 4.5 & 0 & 11.8 & 5.2 & 0 & 0 & Bal \\
\hline
\end{tabular}

Table 3. Efficiency indices of alloys.

\begin{tabular}{|c|c|c|c|c|c|c|}
\hline \multirow{2}{*}{ Alloy } & \multicolumn{2}{|c|}{$\sigma_{100}^{1000}, \mathrm{MPa}$} & \multicolumn{2}{c|}{$T_{\text {Solvus }}$} & \multicolumn{2}{c|}{$V_{\gamma^{\prime}}$} \\
\cline { 2 - 7 } & Model & Experiment & Model & Experiment & Model & Experiment \\
\hline SLGS-1 & 245 & 265 & 1304 & 1302 & 68.8 & 69.4 \\
\hline SLGS-3 & 337 & 345 & 1330 & 1330 & 68.1 & 69.2 \\
\hline
\end{tabular}

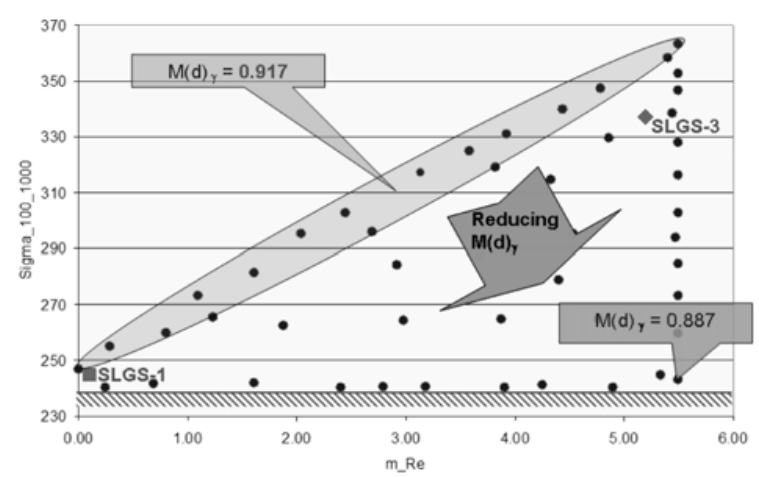

Figure 4. Results of optimization - the set of Pareto-optimum solutions.

- $\sigma_{100}^{1000}-$ maximization, applying constraint on its minimum value of $240 \mathrm{MPa}$.

- $M(\bar{d})_{\gamma}-$ minimization, applying constraint on its maximum value of 0.917 .

- Mass fraction of rhenium $m_{R e}$ - minimization.

Constraints were applied to values of thermodynamic and concentration parameters, and also to sum of doping elements in $\gamma$ phase.

The fraction of ruthenium was set to zero. The task was solved in multiobjective statement, assuming determination of Pareto set of 50 points. As known, solution point is Pareto-optimum if no other solution exists (within the same task statement), being better by one efficiency index and simultaneously no worse in others indices. That means that solution points correspond to different degrees of optimum compromise between several objectives, because improving one objective assumes worsening of others.

\section{Results of optimization}

50 Pareto-optimum solutions, obtained as the result of multiobjective optimization task solution, are shown in Fig. 4. Each solution point represents unique (within the Pareto set) composition of an alloy and properties, corresponding to it.

From the point of view of our aspiration to improve simultaneously 3 indicators of efficiency each solution provides the optimum compromise between all indicators. Lets discuss in more details Fig. 4, where the oval outlines the subarea with the worst values of $M(\bar{d})_{\gamma}$.

Within this subarea the "exchange" between rhenium mass fraction and high-temperature long-term strength $\sigma_{100}^{1000}$ is clearly observed: rise of $\sigma_{100}^{1000}$ is possible only at the expense of rise in alloy price due to increasing doping

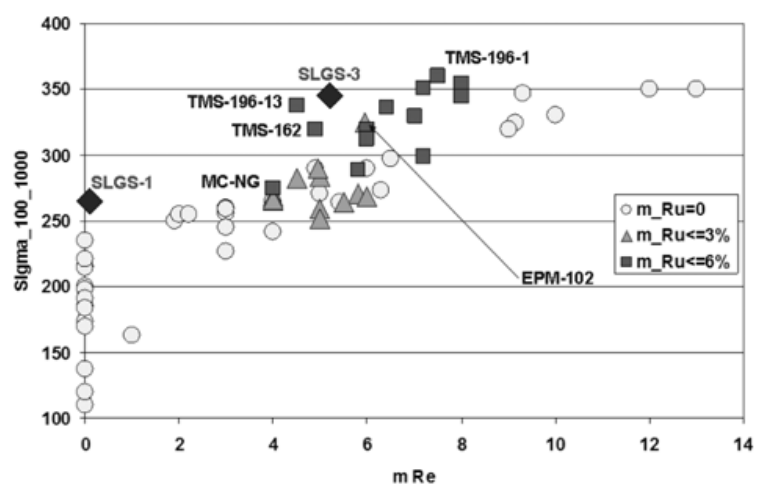

Figure 5. The place of SLGS-1 and SLGS-3 among existing superalloys.

with rhenium. Moving towards the right and down out of the specified subarea leads to simultaneous deterioration of long-term strength and increasing of $m_{R e}$, however the indicator $M(\bar{d})_{\gamma}$ improves. In the right bottom corner of the plot the point $M(\bar{d})_{\gamma}=0.887$ is signed, it is the best according to $M(\bar{d})_{\gamma}$, but the worst according to both $m_{R e}$ and $\sigma_{100}^{1000}$

Summarizing the obtained results, we should note that the presented technology of search for the composition of alloy demonstrated its applicability. Within the limits of trust to MM of heat resisting alloy, the application of numerical multiobjective optimization allows to find those solutions, which are extremely achievable and provide the optimum compromise between several contradicting requirements.

Having available the set of Pareto-optimum solutions, the engineer-researcher can make reasonable choice of composition of the alloy with desirable properties, and to have thus strong reasons to believe that the choice is optimum.

In Fig. 4 the square symbols outline new alloys SLGS1 and SLGS-3 [7], which possess unique combinations of properties (see Fig. 4 and Tables 2 and 3). In particular, SLGS-1 provides the long durability at the level of the best alloys of 2-nd generation, and almost does not contain rhenium. The indicator of high-temperature long-term strength of SLGS-3 achieves the level of last generation superalloys, and is provided without doping the alloy with ruthenium. No TCP-phases were observed in microstructure of both alloys.

Figure 5 demonstrates the dependence of long-term strength at $1000^{\circ} \mathrm{C}$ on mass fraction of rhenium for existing superalloys. This figure characterizes the world trend of creating nickel materials with ultimate properties - raise of high-temperature strength is achieved by 
increasing mass fraction of rhenium (ruthenium is also added last decade to improve the stability and prevent TCP-phases formation). However, points of SLGS-1 and SLGS-3 lay $40 \ldots 50 \mathrm{MPa}$ higher as compared to nonruthenium alloys, having corresponding mass fraction of rhenium. Absence of expensive ruthenium and low contents of extremely scarce and rather expensive rhenium is considerably beneficial to the cost of new alloys $(1.4 \ldots 2$ times for SLGS-3 and 8 times for SLGS-1) as compared to existing alloys of the same category.

\section{Conclusion}

The presented technology of development of optimized composition of heat resisting alloy demonstrated its working capacity. Its distinctive feature is possibility to predict extremely achievable properties of alloy and to provide the desirable compromise between several inconsistent requirements. The computer model is opened from the point of view of adding new points into the source database and getting more accurate approximation functions in order to increase the efficiency of its application in a combination with procedure of numerical optimization. The technology provides automated search for optimum compositions of the alloys according to the set of strength, structure, thermodynamic and other indices, defined by researcher.

\section{References}

[1] P. Caron, "High $\gamma^{\prime}$-solvus new generation nickelbased superalloys for single crystal turbine blade application”, Superalloys, A publ of the Minerals, Met. and Mater. Soc., Champion (Pennsylvania), (2000), 737-746

[2] M. Morinaga, N. Yukawa, H. Adachi, H. Ezaki, "New PHACOMP and its applications to alloy design", Superalloys 1984, ed. M. Gell et al. (Warrendale, PA, USA: the metallurgical society of AIME (1984) 523532)

[3] N. Yukawa, M. Morinaga, H. Ezaki, Y. Murata, Alloy design of superalloys by the d-electrons concept (paper presented at "High temp, alloys for gas turbines and other applications" Conference, Liege (Belgium), (1986), 935-944

[4] V. Nikitin, Corrosion and Protection of Gas Turbines Blades (Leningrad, Mashinostroenie (1987)

[5] F. Jurecka, "Robust Design Optimization Based on Metamodeling Techniques". Thesis of Doctoral Dissertation, Engineering, Technical University of Munchen (2007)

[6] I. Egorov, G. Kretinin, I. Leshchenko and S. Kuptzov. "IOSO NM Optimization Toolkit - Novel Software for Multidisciplinary Optimization" Proceedings of $6^{\text {th }}$ World Congress on Structural and Multidisciplinary Optimization, Rio de Janeiro, May 30-June 3 (2005)

[7] Yu. Shmotin, A. Logunov, D. Danilov, I. Leshchenko. Development of Economically Doped Heat-Resistant Nickel Single-Crystal Superalloys for Blades of Perspective Gas Turbine Engines (DVD-Proceedings of $8^{\text {th }}$ Pacific Rim International Congress on Advanced Materials and Processing (PRICM-8), ISBN 978-0470-94309-0, Wiley, TMS, 2013) 